\title{
Informe sobre el I Congreso Científico de Judo Escuela De Frutos - Universidad Miguel Hernández con Campo de Entrenamiento
}

\author{
Juan José PULIDO GONZÁLEZ*
}

Universidad de Extremadura. Facultad de Ciencias del Deporte (España)

Recepción: 25/11/2013; Aceptación: 05/12/2013; Publicación: 09/12/2013.

\section{Resumen}

El presente informe resume lo acontecido en el I Congreso Científico de Judo Escuela De Frutos-Universidad Miguel Hernández, celebrado en San Juan de Alicante (España) entre los días 27 y 30 de junio de 2013. Se llevaron a cabo 3 ponencias plenarias en aula, 12 comunicaciones orales, una mesa redonda, 2 talleres teórico prácticos sobre tatami, un taller de auto-vendaje funcional para judocas, 2 clases magistrales de judo sobre tatami y 3 sesiones de randori. Este congreso fue un evento novedoso en el que se citaron personalidades del mundo del judo a nivel internacional junto con científicos provenientes de casi toda la geografía española preocupados por esta disciplina deportiva. El evento cumplió el objetivo de acercar la ciencia a los profesionales del judo y viceversa. Se dieron cita un total de 112 participantes.

Palabras clave: Judo; investigación científica; congresos; entrenamiento; rendimiento.

Report about the $1^{\text {st }}$ De Frutos - Universidad Miguel Hernández Judo Scientific Congress with Training Camp

\begin{abstract}
The current report resumes the $1^{\text {st }}$ De FrutosUniversidad Miguel Hernández Judo Scientific Congress, held in San Juan de Alicante (Spain) from the $27^{\text {th }}$ to the $30^{\text {th }}$ of June in 2013. Three plenary sessions in classroom, 12 oral communications, a round - table, 2 theoretical practical workshops on tatami, and 3 randori sessions were developed. This congress was a new event where judo personalities at the international level as well as scientists from the whole Spanish geography interested in this field assisted. The event complied the aim to approach the science to judo professionals and vice versa. A total amount of 112 participants were in the congress.
\end{abstract}

Keywords: Judo; scientific research; congress; training; performance.
Resumo sobre o I Congresso Científico de Judo Escola De Frutos - Universidade Miguel Hernández com Campo de Treinos

\section{Resumo}

O presente texto apresenta o resumo do I Congresso Científico de Judo Escola De Frutos - Universidade Miguel Hernández, realizado em San Juan de Alicante (Espanha), entre os dias 27 e 30 de junho de 2013. Em termos de realização e de organização, o Congresso contou com 3 sessões plenárias em aula, 12 comunicações orais, 1 mesa-redonda, 2 workshops teóricopráticos no tatami, um workshop de auto-vendagem funcional para judocas, 2 classes magistrais de judo sobre o tatami, e 3 sessões de randori. Foi um evento pioneiro, onde se citaram personalidades do mundo do judo internacional com os académicos e científicos provenientes de quase todo o território espanhol, preocupados por esta disciplina desportiva. 0 evento cumpriu o objetivo de aproximar a ciência e os profissionais do judo e vice-versa. 0 evento contou com a participação de 112 participantes.

Palavras-chave: Judo; investigação científica; congressos; treino; rendimento.

\section{1.- Introducción}

El judo como disciplina deportiva muestra un notable grado de madurez en España, país en el que se han formado grandes campeones a nivel tanto europeo como mundial (Pérez, GutiérrezGarcía, y Calvo, 2005). Este deporte cuenta con un importante cuerpo de conocimiento práctico debido al gran número de practicantes y maestros preocupados por su desarrollo. Asimismo, está

\footnotetext{
*E-mail: jjpulido@unex.es. Dirección: Facultad de Ciencias del Deporte. Avda. de la Universidad, s/n. 10071. Cáceres, España.
} 
afianzado en el ámbito académico a través de las numerosas facultades de Ciencias de la Actividad Física y el Deporte donde se imparten asignaturas centradas total o parcialmente en el judo (Gómes y Avelar-Rosa, 2012). Es también en el ámbito universitario donde han surgido, particularmente desde la década de 1990, iniciativas individuales y grupales de trabajos de investigación que han abordado el estudio del judo desde algunas de sus múltiples facetas (Gutiérrez-García y PérezGutiérrez, 2009).

Hasta el momento, se han llevado a cabo en nuestro país múltiples concentraciones deportivas de judo con un formato cuyo fin es el entrenamiento de deportistas sobre el tatami en la búsqueda de aumentar su rendimiento deportivo a través de métodos tradicionales de entrenamiento. Algunas de estas concentraciones se han consolidado como referentes a nivel mundial y reúnen cada año a las mejores selecciones nacionales del mundo, como es el caso del Training Camp organizado por la Real Federación Española de Judo y D.A. y que se celebra en Castelldefels todos los veranos desde hace años. También es habitual la celebración en España de eventos científicos (congresos, jornadas, simposios, etc.) relacionados con la actividad física y el deporte. Específicamente, se han celebrado eventos sobre deportes y actividades de lucha (véanse e.g., Amador, Castro, y Álamo, 1997; Amador, Castro, Álamo, Dopico, e Iglesias, 2004), y la Federación Madrileña de Judo ha venido organizando en años recientes un congreso para profesores de judo en el que se han presentado trabajos de carácter académico (véanse e.g., Federación Madrileña de Judo y Deportes Asociados, 2010, 2011). Con todo, han sido muy escasos los eventos de corte científico con el judo como objetivo central.

En este contexto de progresivo desarrollo del judo, el I Congreso de Judo Escuela De Frutos Universidad Miguel Hernández (CCJEDF-UMH) se ideó como una novedosa iniciativa para acercar a los diferentes grupos de investigación sobre judo existentes en nuestro país, con el propósito de que afianzasen algunas líneas de actuación conjunta para el futuro próximo. Con ello se pretendería fomentar proyectos intergrupales que permitiesen emprender trabajos de una dimensión mayor de la que habitualmente pueden realizar grupos individuales de forma aislada. Asimismo, un segundo objetivo fundamental fue transferir el conocimiento generado desde el ámbito académico a aquellos más implicados en la práctica, esto es, profesores y judocas, por la convicción de que el modo de obtener el conocimiento para progresar pasa cada vez más por la ciencia. Y es que, como sucede en muchos otros ámbitos, a menudo existe una distancia entre la ciencia y el entorno del judoca que entrena cada día en el tatami. Tan difícil es para profesores, entrenadores y deportistas contar con información proveniente de la ciencia y específica de su deporte, como para los científicos acceder a muestras de judocas con el fin de realizar sus investigaciones. Ante esta situación, los perjudicados son todos los que realmente están interesados en el judo.

El I Congreso de Judo Escuela De Frutos - UMH se celebró en San Juan de Alicante (España) entre los días 27 y 30 de junio de 2013, bajo la responsabilidad principal del Dr. Carlos Montero Carretero, profesor de la UMH y Director Técnico de la Escuela De Frutos. Su celebración fue posible gracias a la colaboración del Ayuntamiento de San Juan de Alicante, y particularmente de su concejal de deportes D. Víctor García Berenguer y del alcalde de la localidad D. Manuel Aracil Llorens, que cedieron las instalaciones municipales para el desarrollo de las diferentes actividades. Cabe destacar asimismo el apoyo en la organización y el desarrollo de trabajos por parte del Centro de Investigación del Deporte de la UMH de Elche, así como del grupo de trabajo de Escuela De Frutos-Ozone de Alicante. Herycor, empresa especializada en material de fisioterapia con sede en Elche (Alicante) hizo posible que se desarrollaran los talleres de vendaje funcional. Un total de 112 participantes, en su mayoría de España, se dieron cita en el congreso. Seguidamente se realizará una breve síntesis de las diversas actividades que conformaron el mismo.

\section{2.- Desarrollo}

\section{1.- Ponencias plenarias}

Se desarrollaron tres ponencias plenarias. La primera, titulada "Entrenamiento de la fuerza en judocas. Análisis de los diferentes modelos de planificación" fue realizada por el Dr. Juan Bonitch Góngora. En ella se pusieron de manifiesto las principales aplicaciones que se derivan de los hallazgos obtenidos en sus recientes investigaciones sobre la temática (Bonitch-Domínguez, 
Bonitch-Góngora, Padial, y Feriche, 2010; Bonitch-Góngora et al., 2007; Bonitch-Góngora, BonitchDomínguez, Padial, y Feriche, 2012), destacando la importancia que tienen determinadas manifestaciones de fuerza en el judoca de competición y su relación con las acciones características en este deporte.

La segunda ponencia plenaria titulada "Claves para motivar en judo", a cargo del Dr. Carlos Montero Carretero, analizó la importancia de la motivación en términos generales y dio a conocer las teorías más importantes que explican el comportamiento de los seres humanos a través de sus motivaciones, centrándose fundamentalmente en la Teoría de la Autodeterminación (Deci y Ryan, 1985; Ryan y Deci, 2000) y más concretamente en su aplicación con judocas. Se presentó oficialmente una guía sobre estrategias motivacionales en judo, actualmente en proceso de edición por parte de la Federación Gallega de Judo, que se espera sea una herramienta útil para profesores y entrenadores.

Finalmente, el subcampeón del mundo y campeón de Europa Sugoi Uriarte analizó en la tercera ponencia plenaria, titulada "Estudio táctico: análisis de rivales en judo", la importancia del componente táctico en este deporte. Uriarte mostró su metodología de análisis de rivales aportando una visión meticulosa sobre la importancia que tiene prestar especial atención a los pequeños detalles en esta tarea.

\section{2.- Talleres teórico-prácticos}

El modelo organizativo adoptado en el congreso (congreso + campo de entrenamiento) permitió que los asistentes vieran cómo se ponían en práctica lo mostrado en las dos primeras ponencias teóricas expuestas con anterioridad. Así, en el primer taller titulado "Entrenamiento integrado de la fuerza en judo" el Dr. Bonitch mostró diferentes ejercicios sobre el tatami para el desarrollo de fuerza elástico reactiva, explosiva e isométrica, justificando en cada caso su utilización. Los entrenadores presentes pudieron resolver sus dudas en el terreno práctico y observar directamente cómo los conocimientos que aporta la ciencia a menudo son fácilmente trasferibles al trabajo diario. Por su parte, en el segundo taller práctico, titulado "Estrategias motivacionales en judo" consistió en el análisis de una segunda sesión de entrenamiento de fuerza en la que los profesores aprendieron a implementar algunas estrategias motivacionales, consistentes fundamentalmente en que los deportistas se sintieran más autónomos, competentes y bien relacionados con los demás durante las clases.

\section{3.- Comunicaciones orales}

Se presentaron un total de 12 comunicaciones orales, moderadas por el Dr. Eduardo Cervelló Gimeno, Decano de la Facultad Socio Sanitaria y Director del Centro de Investigación de la Universidad Miguel Hernández de Elche.

En primer lugar, se mostraron algunos hallazgos referentes a la estabilidad del raquis, fuerza y resistencia de la musculatura del tronco en judocas, de la mano del Grupo de Investigación en Biomecánica para la Salud y el Rendimiento Deportivo (BIOMEC) que dirige el Dr. Francisco José Vera García de la UMH de Elche. Desde este mismo grupo se presentó un trabajo dirigido por el Dr. José Luis López Elvira, en el que se pusieron de manifiesto algunas características de los impactos que se producen al realizar la técnica seoi-otoshi por parte de judocas de diferentes niveles de dominio y grado.

El grupo de investigación que dirige el Dr. Enrique Roche, del Instituto de Bioingeniería de la UMH, presentó cuatro comunicaciones en las que se contextualizó el modo en que los judocas de competición abordan las estrategias para dar el peso, enseñando además los resultados de algunos estudios que muestran las ventajas de hacerlo bajo la supervisión de profesionales que puedan controlar correctamente la ingesta de macronutrientes en función del gasto calórico estimado de los judocas. Además se presentó un cuestionario que puede resultar de utilidad para los profesores de judo a la hora de detectar posibles trastornos de conducta alimentaria, aún en desarrollo.

Por otra parte, se presentó en un estudio piloto desarrollado por miembros del GIAFIS (Grupo de Investigación en Acondicionamiento Físico Saludable) de la UMH, dirigido por el Dr. 
Manuel Moya, sobre los efectos de la ingesta de beta alanina sobre el rendimiento de judocas de competición. Si bien la muestra utilizada en este estudio fue muy escasa, los resultados aportaron cierta información relevante al respecto, estando en consonancia con los resultados obtenidos en otros estudios de diferentes disciplinas deportivas.

Se presentaron dos comunicaciones por investigadores externos a la UMH. La Dra. Raquel Hernández, entrenadora de judo en la provincia de Cáceres, analizó algunas de las consecuencias de los recientes cambios reglamentarios en judo en lo referente a la estructura temporal del combate en categoría sénior. Por otro lado, desde la Universidad de Granada, se expusieron por parte de Dña. Filipa Almeida los datos referentes a estudios que han analizado la fuerza isométrica de prensión manual en judocas.

Finalmente, los alumnos de la UMH P. Santis-Mandiola, M. Segura-Ibáñez, E. Torres-Romero y A. J. Soler-Aguinaga mostraron las posibles aportaciones que puede suponer el empleo de una aplicación móvil (FATIGUO), creada por ellos mismos, a la hora de cuantificar la fatiga producida por las cargas de entrenamiento a las que se someten los judocas, a través del uso de cuestionarios de corte psicológico.

\section{4.- Lecciones magistrales de judo}

Las lecciones magistrales sobre el tatami del japonés Go Tsunoda, asesor en el entrenamiento de judocas de selecciones nacionales de Gran Bretaña y Portugal, permitieron a los asistentes ver y practicar técnicas de desplazamiento y proyección de una escuela poco vista en nuestro país. Más de cien judocas presentes en estas sesiones 'aprendieron' los fundamentos técnicos del judo que, en opinión del maestro Tsunoda, son olvidados frecuentemente por los propios profesores de judo.

\section{5.- Otras actividades}

Complementariamente a las actividades ya descritas se desarrolló una mesa redonda titulada "Estrategias en la gestión del judo a diferentes niveles", en la que los ponentes D. Mario Muzas (Presidente de la Federación Gallega de Judo - FGJ), D. Eduardo Galán (Director del Departamento de Imagen y Difusión de la FGJ), el Dr. Vicente Carratalá (Director de la Escuela Federativa Nacional de la Real Federación Española de Judo y D.A.) mostraron algunas perspectivas de gestión relacionada con el judo a nivel federativo y con los clubes privados. Los ponentes insistieron en la importancia de optimizar recursos y, sobre todo, de elaborar estrategias concretas sobre una estructura organizada basada en el trabajo de equipo, tomando como ejemplo el caso de la FGJ. Por su parte, el Dr. Carratalá mostró la capacidad que tiene un club para influir en el entorno más próximo, a través de la herramienta educativa y recreativa que supone el judo.

Asimismo, el fisioterapeuta D. Rafael Gomis desarrolló un taller práctico sobre auto vendajes en judo que pretendió enseñar a judocas y entrenadores a resolver determinadas situaciones de lesión que suelen producirse durante la práctica. Por último, el programa contó con cuatro sesiones de randori orientadas a fomentar el conocimiento mutuo y el intercambio de conocimientos prácticos por parte de los judocas asistentes al campo de entrenamiento.

\section{3.- Conclusiones}

El I Congreso de Judo Escuela De Frutos - UMH supuso una iniciativa que previsiblemente tendrá continuidad en un futuro próximo. La fórmula mixta adoptada permitió el intercambio entre los participantes más interesados en el aspecto académico del estudio del judo y aquellos más orientados a su vertiente más práctica, y podría ser interesante mantener esta idea en el futuro para que ambos colectivos lograsen un mutuo beneficio. Desde la perspectiva académica, la diversidad de trabajos presentados refleja el interés que despierta la investigación sobre judo, y sería deseable que más grupos de investigación e investigadores de otras universidades y centros de investigación españoles y de otros países participasen en las siguientes ediciones del congreso. 


\section{Agradecimientos}

Este trabajo ha sido realizado gracias a la financiación y colaboración del Consejo Superior de Deportes, mediante la convocatoria de Ayudas a Universidades Públicas y Privadas, Entidades Públicas y Entidades sin ánimo de lucro para la realización de Proyectos de investigación, estudios, organización de actos científicos y publicaciones periódicas en áreas de interés deportivo (170/UPB10/12).

\section{Referencias}

Amador, F., Castro, U., \& Álamo, J. M. (Eds.) (1997). Luchas, deportes de combate y juegos tradicionales. Madrid: Gymnos.

Amador, F., Castro, U., Álamo, J. M., Dopico, J. \& Iglesias, E. (Eds.) (2004). Dimensión histórica, cultural y deportiva de las luchas. Gran Canaria: Cabildo Insular de Fuerteventura y ACCEDEL.

Bonitch-Domínguez, J., Bonitch-Góngora, J., Padial, P., \& Feriche, B. (2010). Changes in the peak legpower induced by successive judo bouts and their relationship to lactate production. Journal of Sports Sciences, 28, 1527-1534.

Bonitch-Góngora, J., Bonitch-Domínguez, J., Feriche, B., Chirosa, I., Sánchez, C., Granados, M.A., García, Z., Pintor, M.A., \& Padial, P. (2007). Análisis del comportamiento de la resistencia a la fuerza isométrica máxima de la musculatura prensora del antebrazo en judokas. Archivos de Medicina, 24(121), 358.

Bonitch-Góngora, J., Bonitch-Domínguez, J., Padial, P., \& Feriche, B. (2012). The effect of lactate concentration on the handgrip strength during judo bouts. Journal of Strength and Conditioning Research, 26(7), 1863-1871.

Deci, E.L., \& Ryan, R.M. (1985). Intrinsic motivation and self determination in human behavior. New York: Plenum Press.

Federación Madrileña de Judo y Deportes Asociados (Ed.) (2010). Judo: Una visión diversa (Actas del I Congreso de la Federación Madrileña de Judo). Madrid: Visión Libros.

Federación Madrileña de Judo y Deportes Asociados (Ed.) (2011). Judo: Una visión diversa II. Madrid: Visión Libros.

Gomes, M. S. P. \& Avelar-Rosa, B. (2012). Martial arts and combat sports in Physical Education and Sport Sciences Degrees.A comparative study of Brazil, France, Portugal, and Spain. INYO: The Journal of Alternative Perspectives on the Martial Arts and Sciences, 12(1), 13-28.

Gutiérrez-García, C \& Pérez Gutiérrez, M. (2009). Study on scientific production in martial arts in Spain from 1990 to present. En W. J. Cynarsky, Martial Arts and Combat Sports - Humanistic Outlook (pp. 90-115). Rzeszów: Wydawnictwo Uniwersytetu Rzeszowkiego.

Pérez, D., Gutiérrez-García, C., \& Calvo, R. (2005, Septiembre). Evolución del judo de élite español: una perspectiva histórica. CD de actas del IV International Judo Federation. World Judo Conference, El Cairo.

Ryan, R.M., \& Deci, E.L. (2000). Self-determination theory and the facilitation of intrinsic motivation, social development, and well being. American Psychologist, 55, 68-78.

\section{Datos biográficos de los autores}

Juan José Pulido González es Licenciado en Ciencias de la Actividad Física y el Deporte (CCAFD) por la Universidad de Extremadura. Posee el Máster Universitario en Investigación en Ciencias Sociales y Jurídicas con Especialidad en CCAFD, así como el Máster Universitario en Formación del Profesorado en Educación Secundaria. Actualmente está realizando el doctorado y es becario FPI (Consejería de Empleo, Empresa e Innovación - Gobierno de Extremadura) de investigación del grupo Análisis Didáctico y Comportamental del Deporte. 